\title{
Altered CD94/NKG2A and perforin expression reduce the cytotoxic activity in malignant pleural effusions
}

\author{
Elisabetta Pace ${ }^{a, *}$, Caterina Di Sano ${ }^{a}$, Maria Ferraro ${ }^{a}$, Annalisa Tipa ${ }^{a, b}$, Dario Olivieri ${ }^{b}$,

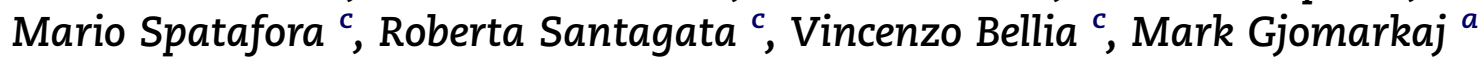 \\ a Istituto di Biomedicina e Immunologia Molecolare, Unità di Immunopatologia e Farmacologia Clinica e Sperimentale dell'Apparato \\ respiratorio, Consiglio Nazionale delle Ricerche, Palermo, Italy \\ ${ }^{\mathrm{b}}$ Dipartimento di Scienze Cliniche, Sezione di Malattie Respiratorie, Università di Parma, Parma, Italy \\ ${ }^{c}$ Dipartimento Biomedico di Medicina Interna e Specialistica, Sezione di Pneumologia, Università di Palermo, Palermo, Italy
}

\section{A R T I C L E I N F O}

Article history:

Received 15 June 2010

Received in revised form 16 August

2010

Accepted 2 September 2010

Keywords:

Lymphocytes

Cancer

Malignant pleural effusions

\section{A B S T R A C T}

CD94/NKG2A is an inhibitory receptor expressed by NK cells and cytotoxic lymphocytes and, upon activation by HLA-E, downregulates the cytolytic activities of these cells thus representing a tumour immune escape mechanism.

This study was aimed at assessing whether cytotoxic lymphocytes (CD8+) and NK cells from malignant pleural effusions have a deregulated expression of CD94/NKG2A.

The expression of membrane CD94/NKG2A and perforin was evaluated by flow-cytometry in CD8+ and NK cells from pleural effusions and autologous peripheral blood of cancer $(n=19)$ and congestive heart failure (CHF) $(n=11)$ patients. Intracellular CD94/NKG2A expression was evaluated by flow-cytometry in pleural effusion CD8+ and NK cells from cancer patients $(n=10)$. Cytotoxic activity against cancer cells exerted by pleural and autologous peripheral blood T lymphocytes from cancer patients was assessed by flow-cytometry assay.

Pleural CD8+ from cancer patients showed a reduced expression of membrane CD94/ NKG2A and perforin when compared to autologous peripheral blood and CHF pleural effusions. Reduced numbers of NK cells were present in pleural effusions from both cancer and CHF patients. Pleural NK from cancer patients showed a reduced expression of membrane CD94/NKG2A and perforin when compared to autologous peripheral blood. Pleural T lymphocytes from cancer patients exhibited a reduced cytotoxic activity against cancer cells when compared to autologous peripheral blood $\mathrm{T}$ lymphocytes. The intracellular expression of CD94/NKG2A in CD8+ and NK cells from cancer patients was higher than membrane expression.

In conclusion, this study provides compelling evidences of new mechanisms underlying the reduced host defence against cancer within the pleural space.

(C) 2010 Elsevier Ltd. All rights reserved.

\section{Introduction}

Malignant pleural effusions are mostly due to metastatic involvement of the pleural compartment by heterogeneous primaries and recur in $15 \%$ of patients who die with malignancies and account for up to $50 \%$ of the exudates in many clinical series. ${ }^{1}$ Exudative malignant pleural effusions are characterised by the accumulation of fluid enriched in proteins and by the recruitment of elevated numbers of mononuclear cells into the pleural space. ${ }^{2}$ Host defence

\footnotetext{
* Corresponding author: Address: Istituto di Biomedicina e Immunologia Molecolare, Consiglio Nazionale delle Ricerche, Via Ugo La Malfa, 153, 90146 Palermo, Italy. Tel.: +39 091680 9148; fax: +39 0916809122.

E-mail address: pace@ibim.cnr.it (E. Pace).
}

0959-8049/\$ - see front matter @ 2010 Elsevier Ltd. All rights reserved.

doi:10.1016/j.ejca.2010.09.001 
mechanisms against tumour cells are mainly related to the activity of CD8+ cytotoxic T lymphocytes (CTL) and natural killer (NK) cells. ${ }^{3}$ CD8+ CTL exert their cytotoxic activity on tumour cells by releasing toxic mediators, such as perforin and granulysin ${ }^{4}$ or by inducing cell apoptosis via FAS/FAS ligand pathway. ${ }^{5}$ Like CD8+ CTL, NK cells kill tumour target cells without prior sensitisation, relying on directed exocytosis of secretory lysosomes and on activation of FAS/FAS ligand-induced cell death. The magnitude and diversity of CD8 positive CTL- and NK-mediated responses against tumour cells reflect the outcome of a balance between signals of activation and inhibition. An accurate and precise control of this balance is essential for mounting protective anti-tumour responses and for concurrently limiting potential immunopathologic complications. ${ }^{6}$ The CD94/NKG2A heterodimer is an inhibitory receptor expressed by CD8+ CTL and NK cells that, upon activation by HLA-E, downregulates their cytolytic activity against tumour cells. ${ }^{7}$ Although, some studies have shown that pleural effusion mononuclear cells exhibit several functional defects, ${ }^{8}$ limited information is available regarding the expression and the activity of the CD94/NKG2A complex in CD8+ CTL and NK cells isolated from malignant pleural effusions.

The aims of the present study were to determine whether CD8+ CTL and NK cells from malignant pleural effusions have a deregulated expression/activation of CD94/NKG2A and whether this phenomenon may contribute to a reduced cytotoxic activity against cancer cells in the pleural compartment.

\section{Materials and methods}

\subsection{Pleural fluid collection}

Peripheral blood (PB) and pleural fluid (PF) were obtained from patients with congestive heart failure (CHF) $(n=11$; age range $50-80$ years) and cancer ( $n=19$, age range $45-78$ years). Pleural fluids were collected by thoracentesis, before the initiation of chemotherapy. All subjects gave informed written consent and the study was approved by the institutional review board for human studies and was consistent with Helsinki Declaration. Patients were recruited at the Dipartimento di Medicina Interna e Specialistica - Università di Palermo, in Palermo and the Dipartimento di Scienze Cliniche, Sezione di Malattie Respiratorie, Università di Parma, in Parma, Italy.

The effusions were first classified as transudates or exudates by meeting at least one of the criteria described by Light. ${ }^{9}$ Standard clinical, laboratory, and radiological investigations were used to establish the diagnosis for each patient as previously described. ${ }^{10}$ No patients were undergoing antiinflammatory or steroid therapies. The fluids were drawn into polypropylene bags containing heparin (10-20 IU/ml) and were subsequently centrifuged at $400 \mathrm{~g}$ for $10 \mathrm{~min}$.

\subsection{Mononuclear cells}

PB and PF mononuclear cells were isolated by Ficoll-Hypaque (Pharmacia) gradient centrifugation and, after two washes, the cells were suspended in RPMI 1640 tissue culture medium (Invitrogen Life Technologies) supplemented with 10\% heatinactivated foetal calf serum (FCS) (Invitrogen Life Technologies), $2 \mathrm{mM}$ L-glutamine, $20 \mathrm{mM}$ HEPES, $100 \mathrm{U} / \mathrm{ml}$ penicillin,
$100 \mu \mathrm{g} / \mathrm{ml}$ streptomycin, $5 \times 10^{-5} \mathrm{M} 2-\mathrm{ME}$ and $85 \mu \mathrm{g} / \mathrm{ml}$ gentamicin. Purity and viability were tested using trypan blue exclusion.

\subsection{Expression of surface markers}

PB and PF mononuclear cells were washed twice in PBS (containing $1 \%$ FCS and $0.02 \% \mathrm{Na}$ azide) and thereafter stained and subjected to flow cytometric analysis. Suspended cells were stained with FITC-, phycoerythrin-(PE) or phycoerythrin-Cy5 (PE-cy5)-conjugated antibodies against CD3, CD4, CD8, CD19, CD56, CD16 and CD94 [all monoclonal antibodies (MoAb) were from BD PharMingen]. To identify NK cells, PF mononuclear cells were depleted from CD3+ cells using CD3 microbeads (Miltenyi Biotec, ergish Gladbach, Germany) using the MACS cell separation columns, following the manufacturer's instructions. The CD3 depleted cells were then stained using phycoerythrin (PE)-anti CD16 monoclonal antibody and PE-cy5 anti-CD56 (Becton-Dickinson, United States). The CD56+/16+ cells were gated from the total cell population for assessing CD94/NKG2A or perforin expression using FITC conjugated antibodies. To assess CD94/NKG2A expression, a monoclonal antibody (HP-3D9) recognising the dimer Kp43, formed by the association of CD94 with NKG2A (Mingari MC, International Immunology 1887), was used. Cells $\left(10^{5}\right)$ from each sample were analysed using a FACScalibur supported with CellQuest acquisition and data analysis software (Becton Dickinson). The lymphocytes were gated by forward and side scatter.

\subsection{Intracellular staining}

For detection of intracellular perforin or intracellular CD94/ NKG2A, freshly isolated PF and PB cells were surface stained with CD3, CD8 and CD56, in incubation buffer (PBS containing $1 \%$ FCS and $0.1 \% \mathrm{Na}$ azide) for $30 \mathrm{~min}$ at $4{ }^{\circ} \mathrm{C}$. Cells were then washed twice in PBS with $1 \%$ FCS and fixed with PBS containing $4 \%$ paraformaldehyde overnight at $4{ }^{\circ} \mathrm{C}$. Fixation was followed by two washes in permeabilisation buffer with PBS containing $1 \%$ FCS, $0.3 \%$ saponin and $0.1 \% \mathrm{Na}$ azide for $15 \mathrm{~min}$ at $4{ }^{\circ} \mathrm{C}$ and fixed permeabilised cells were stained with antiperforin antibody (BD PharMingen Reagent set). After two more washes in PBS containing 1\% FCS, the cells were analysed by FACSCalibur. Lymphocytes were gated by forward and side scatter and analysis was done on 100,000 acquired events for each sample.

\subsection{Cytotoxicity assay}

In a flow cytometric test effector CD8+ CTL and NK cells from PB and PF from cancer patients $(n=8)$ and from PF from CHF patients $(n=8)$ were mixed with H292 target cells labelled with DiO (3,3'-dioctadecyloxacarbocyanine perchlorate) at effector/target cell ratio of 5:1. Dead cells were stained with propidium iodide and results were expressed as percentage of cytotoxic activity versus baseline.

\subsubsection{Effector cells}

Mononuclear cells were separated from PB and PF from cancer patients and from PF from CHF patients $(n=5)$ by FicollHypaque density gradient centrifugation and adherent cells 
were removed (more than 95\%) by incubation in a glass flask for $60 \mathrm{~min}$ at $37^{\circ} \mathrm{C}$. Non-adherent cells were resuspended in RPMI 1640 medium with 10\% FCS and adjusted to $1 \times 10^{9}$ cells $/$.

\subsubsection{Target cells}

NCI-H292 (mucoepidermoid carcinoma), a Non Small Cell Lung Carcinoma cell line, was maintained in culture in RPMI 1640 medium with $10 \%$ FCS at $37{ }^{\circ} \mathrm{C}$ in a humidified $5 \% \mathrm{CO}_{2}$ chamber. H292 cells were labelled with the stain 3,3'-dioctadecyloxacarbocyanine perchlorate (DiO, Invitrogen, Molecular Probes) for $20 \mathrm{~min}$ of incubation at $37{ }^{\circ} \mathrm{C}$ and $5 \% \mathrm{CO}_{2}$.

\subsubsection{Cell activity assay}

CTL and NK activity against H292 target cells was assessed by a flow-cytometry assay using the DiO membrane dye to stain live H292 cells and propidium iodide (Sigma-Aldrich, St. Louis, MO) nuclear dye to stain dead cells. Unstained effector cells and stained target cells were added to each of six Falcon polystyrene $12 \times 75$ (Becton Dickinson, Mountain View, CA) assay tubes at effector/target ratio of 5:1. Control tubes contained effector and target cells separately. Propidium iodide was added ( $13 \mathrm{mg} /$ tube) and the tubes were incubated in a $5 \% \mathrm{CO}_{2}$ atmosphere at $37^{\circ} \mathrm{C}$ for $2 \mathrm{~h}$. The use of flow-cytometry (FACScalibur D275) in combination with propidium iodide permitted the differentiation of four cell populations: live target cells (upper left), dead target cells (upper right), live effector cells (lower left) and dead effector cells (lower right).

\subsection{Statistics}

Data are expressed as mean counts \pm standard deviation. Sample size for CD94 expression was calculated and was equivalent to $n=13(\alpha=0.05 ;$ power $=0.8$; difference $=3.5$; S.D. = 2.6). The Mann Whitney U-test was used for comparison between patient groups. The paired t test was used for the comparisons of data from cytotoxic assays or for comparing intracellular and membrane expression of CD94/NKG2A in pleural cells from cancer patients. $p<0.05$ was accepted as statistically significant.

\section{Results}

\subsection{CD8+ CTL and NK cells in cancer and in CHF patients}

Altered numbers of NK and CD8+ cells or functional defects of these cell types may contribute to reduce the efficiency of the immune responses against cancer cells. ${ }^{3,4}$ The first step of this study was to assess whether the altered numbers of CD8+ and NK cells were specifically present in malignant PF comparing malignant $\mathrm{PF}$ and $\mathrm{PB}$ from cancer patients with $\mathrm{PF}$ and $\mathrm{PB}$ from CHF patients.

Whilst no significant differences were observed in the percentage of CD8+ CTL between cancer and CHF patients in the two studied compartments (PF and $\mathrm{PB}$ ), the percentage of NK cells was reduced in PF from both cancer and CHF patients in comparison to autologous PB (Table 1).

\subsection{Membrane CD94/NKG2A expression in CD8+ CTL and NK cells of cancer and CHF patients}

CD8+ CTL and NK cells express inhibitory receptors to restrain their 'lethal' behaviour. ${ }^{6}$ Therefore, we assessed whether the expression of the inhibitory receptor CD94/ NKG2A was altered in the total lymphocyte population isolated from malignant $\mathrm{PF}$ comparing malignant $\mathrm{PF}$ and $\mathrm{PB}$ from cancer patients with $\mathrm{PF}$ and $\mathrm{PB}$ from CHF patients.

The membrane expression of CD94/NKG2A was significantly $(p<0.05)$ reduced in malignant $\mathrm{PF}$ in comparison to autologous PB and in comparison to CHF PF (Fig. 1). We further assessed whether this reduced expression of CD94/ NKG2A was related to CD8+ CTL or to NK cells. The expression of CD94/NKG2A by CD8+ CTL was significantly $(p<0.05)$ reduced in malignant $\mathrm{PF}$ in comparison to autologous $\mathrm{PB}$ and in comparison to CHF PF (Fig. 2). The expression of CD94/ NKG2A by NK cells was significantly $(p<0.05)$ reduced in malignant $\mathrm{PF}$ in comparison to autologous $\mathrm{PB}$ but not in comparison to CHF PF (Fig. 3).

\subsection{Perforin expression in CD8 CTL and NK cells of cancer and $\mathrm{CHF}$ patients}

CD8+ CTL and NK cells kill tumour target cells relying on directed exocytosis of secretory lysosomes, including perforin. ${ }^{4}$ Therefore, we assessed whether the expression of perforin was altered in cells obtained from malignant PF by comparing their activity with those of cells from autologous $\mathrm{PB}$ and from $\mathrm{PF}$ and $\mathrm{PB}$ of CHF patients.

The expression of perforin was significantly $(p<0.05)$ reduced in malignant PF in comparison to autologous $\mathrm{PB}$ and in comparison to CHF PF (Fig. 4). Furthermore, the expression of perforin was significantly $(p<0.05)$ reduced in both CD8+ CTL (Fig. 5) and in NK (Fig. 6) cells in malignant PF in comparison to autologous $\mathrm{PB}$.

Table 1 - Phenotype of the cells from PF and PB.

\begin{tabular}{llllll} 
& CD3+ & CD3+CD4+ & CD3+CD8+ & CD3-CD19+ & CD3-CD56+CD16+ \\
\hline Cancer PF & $77 \pm 19$ & $60 \pm 17$ & $17 \pm 6.6$ & $6.9 \pm 5.6$ & $3.4 \pm 3^{*}$ \\
Cancer PB & $57 \pm 25$ & $35.4 \pm 17$ & $19.3 \pm 14$ & $6.8 \pm 5$ & $15 \pm 10$ \\
CHF PF & $75.6 \pm 18$ & $57.5 \pm 20$ & $18.7 \pm 10$ & $3.5 \pm 2.8$ & $5.8 \pm 5^{*}$ \\
CHF PB & $69.5 \pm 19$ & $44.8 \pm 21$ & $26.2 \pm 16$ & $4.8 \pm 2.3$ & $14 \pm 11$ \\
\hline Data are expressed as \% of positive cells. & & & & \\
$*$ &
\end{tabular}



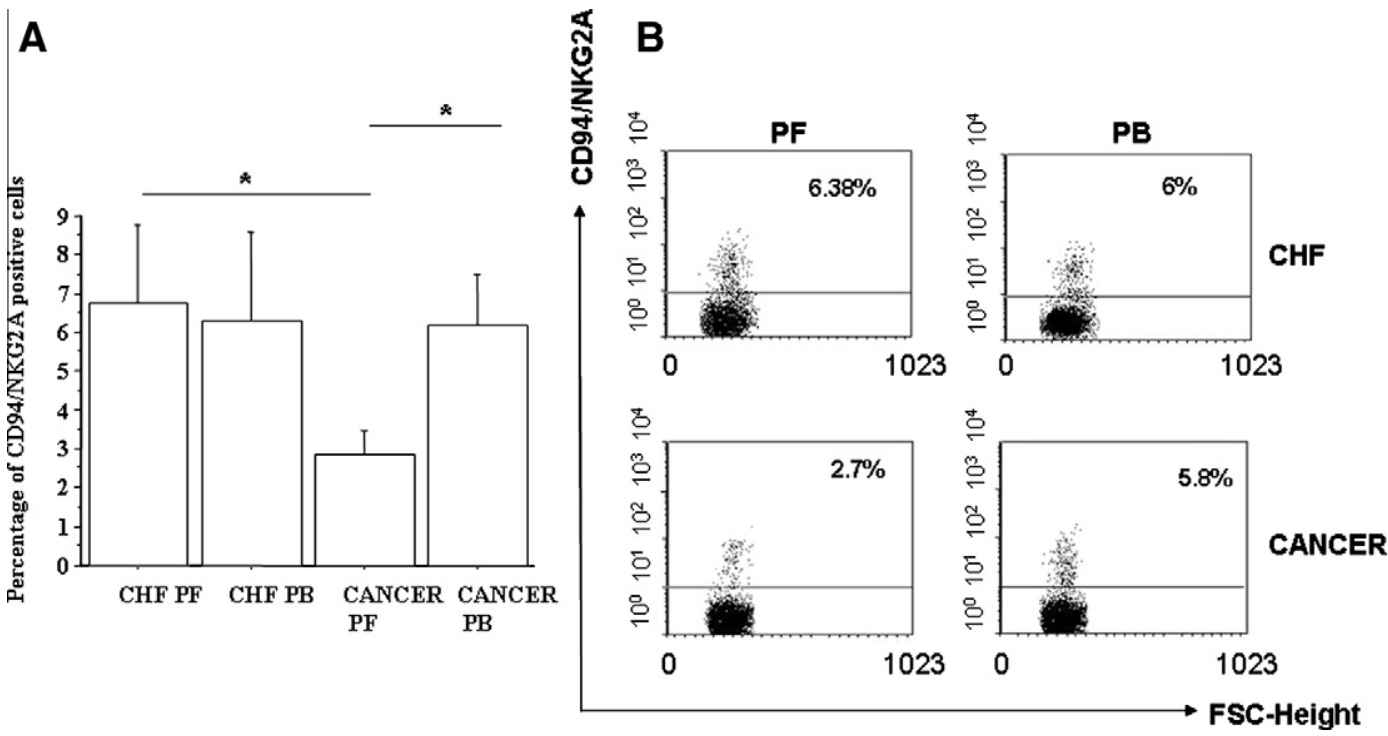

Fig. 1 - CD94/NKG2A expression in cancer and CHF patients. Pleural and peripheral blood mononuclear cells were isolated from malignant $(n=19)$ and from CHF $(n=11)$ pleural fluids and CD94/NKG2A expression was assessed by flow-cytometry in the lymphocytes gated by forward and side scatter (see Section for details) using a FITC conjugated antibody. Data are expressed as percentage of positive cells (mean \pm S.D.) (A). ${ }^{*} p<0.05$ (Mann Whitney U-test). (B) Representative dot plots that show the percentage of total lymphocytes expressing CD94/NKG2A.

3.4. Cytotoxic activity against cancer cells in cancer PF

Since both CD8+ CTL and NK cells present in malignant PF showed reduced expression of perforin when compared to autologous PB and in comparison to CHF PF, we tested their in vitro cytotoxic activity against epithelial cancer cells. In cancer patients, the cytotoxic activity of cells isolated from $\mathrm{PF}$ was reduced when compared to the cytotoxic activity of cells isolated from autologous PB peripheral blood and from CHF PF (Fig. 7).

\subsection{Intracellular CD94/NKG2A expression in CD8 and NK cells of cancer}

Since pleural cells from cancer patients had a reduced membrane expression of the inhibitory receptor CD94/NKG2A but
A

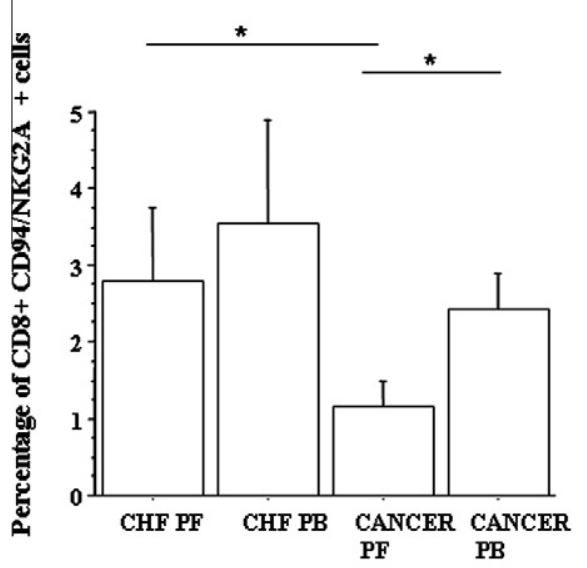

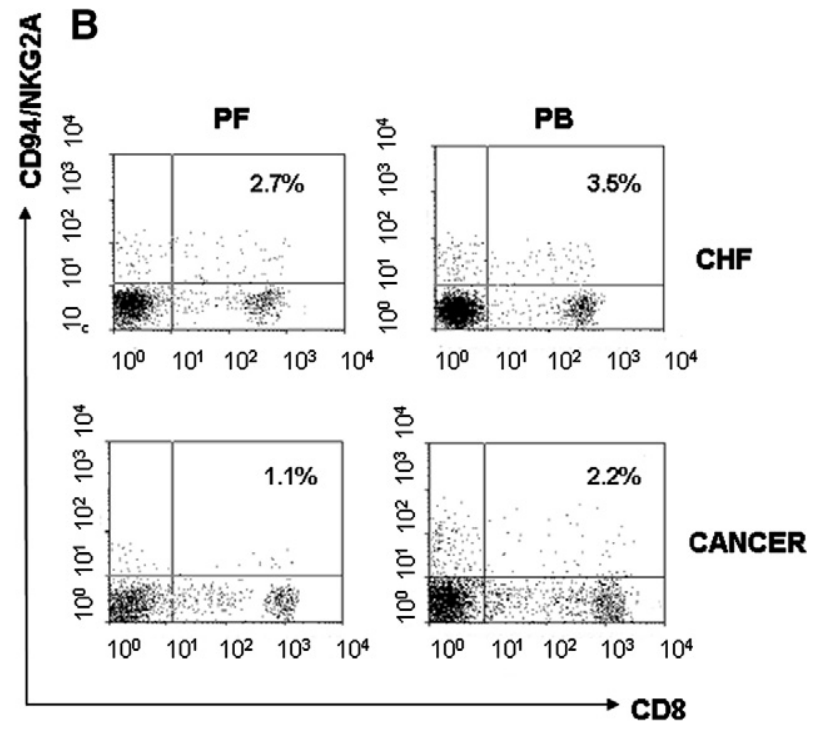

Fig. 2 - CD94/NKG2A expression in CD8+ cells from cancer and CHF patients. Pleural and peripheral blood mononuclear cells were isolated from malignant $(n=19)$ and from CHF $(n=11)$ pleural fluids and CD94/NKG2A expression in CD8+ cells was assessed by flow-cytometry in the lymphocytes gated by forward and side scatter (see Section for details) using a FITC conjugated antibody for CD94/NKG2A and a PE conjugated antibody for CD8. Data are expressed as percentage of positive cells (mean \pm S.D.) (A). * $p<0.05$ (Mann Whitney $U$-test). (B) Representative dot plots that show the percentage of CD8+ cells expressing CD94/NKG2A 

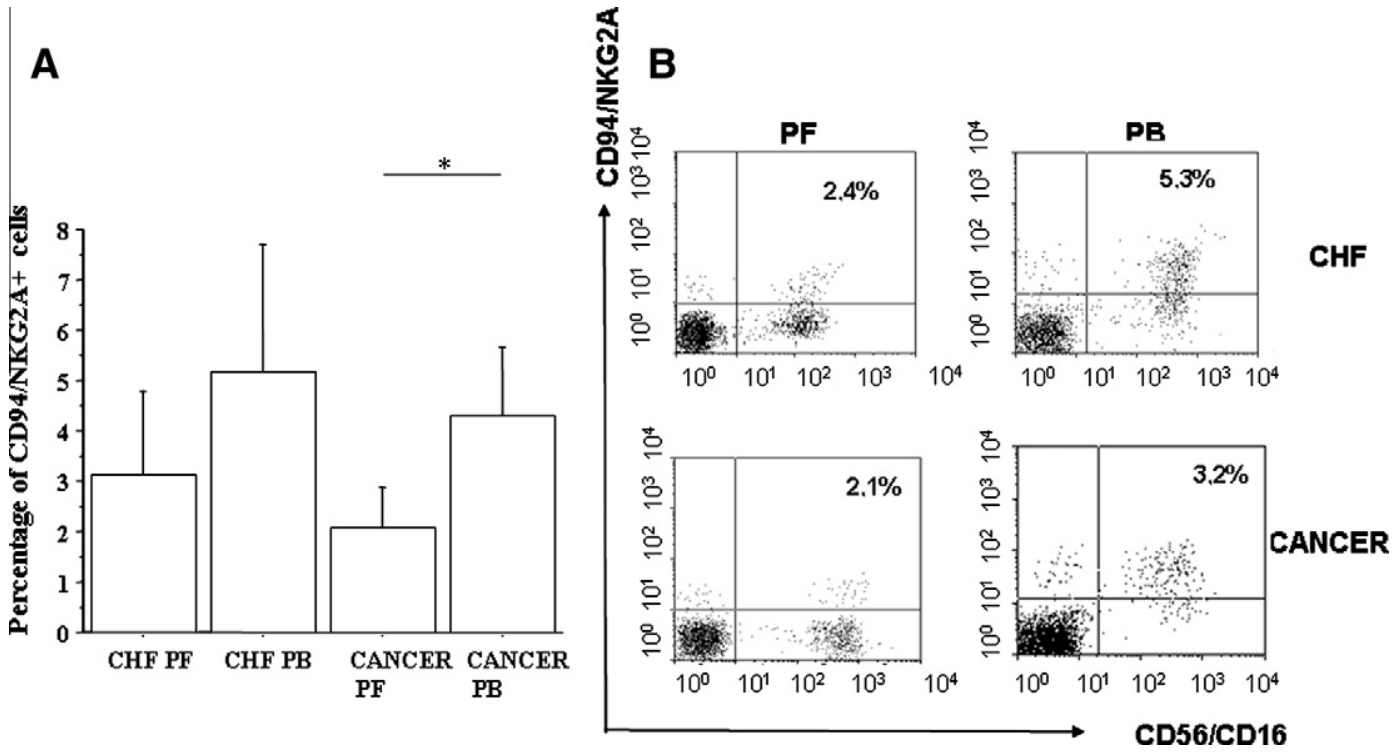

Fig. 3 - CD94/NKG2A expression in CD56+/CD16+ cells from cancer and CHF patients. Pleural and peripheral blood mononuclear cells were isolated from malignant $(n=19)$ and from CHF $(n=11)$ pleural fluids and CD94/NKG2A expression in double positive CD16+/CD56+ (NK) cells was assessed by flow-cytometry (see Section for details) using a FITC conjugated antibody for CD94/NKG2A and a PE-anti CD16 and PE-Cy5 anti-CD56 conjugated antibodies. Data are expressed as percentage of positive cells (mean \pm S.D.) (A). ${ }^{*} p<0.05$ (Mann Whitney U-test). (B) Representative dot plots that show the percentage of CD56/CD16+ cells expressing CD94/NKG2A.

a reduced expression of perforin and a reduced cytotoxic activity against cancer cells and since upon the activation of CD94/NKG2A this receptor is internalised (and therefore no longer on the surface), ${ }^{11}$ we studied the intracellular expression of CD94/NKG2A. In total $\mathrm{T}$ lymphocyte population (Fig. 8A), in CD8+ CTL (Fig. 8B) and in NK cells (Fig. 8C) from malignant $\mathrm{PF}$, the intracellular expression of CD94/NKG2A was significantly $(p<0.05)$ higher than the membrane expression of CD94/NKG2A.

\section{Discussion}

CD8+ CTL and NK cells display effector functions, including the recognition and lysis of infected, stressed, or transformed cells and the production of immunoregulatory cytokines and chemokines. ${ }^{12}$ Tumour microenvironment may be unfavourable for CD8+ CTL and NK cell activity and may contribute to tumour immune escape. Malignant effusions are a relatively easily accessible source of tumour-associated effector

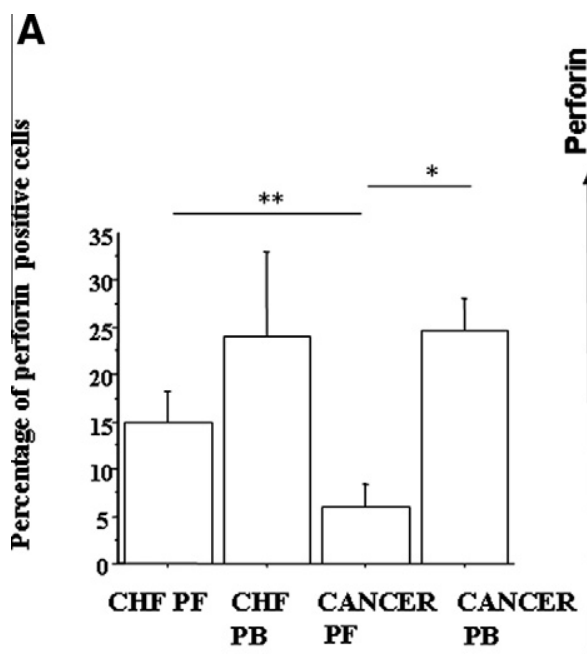

B
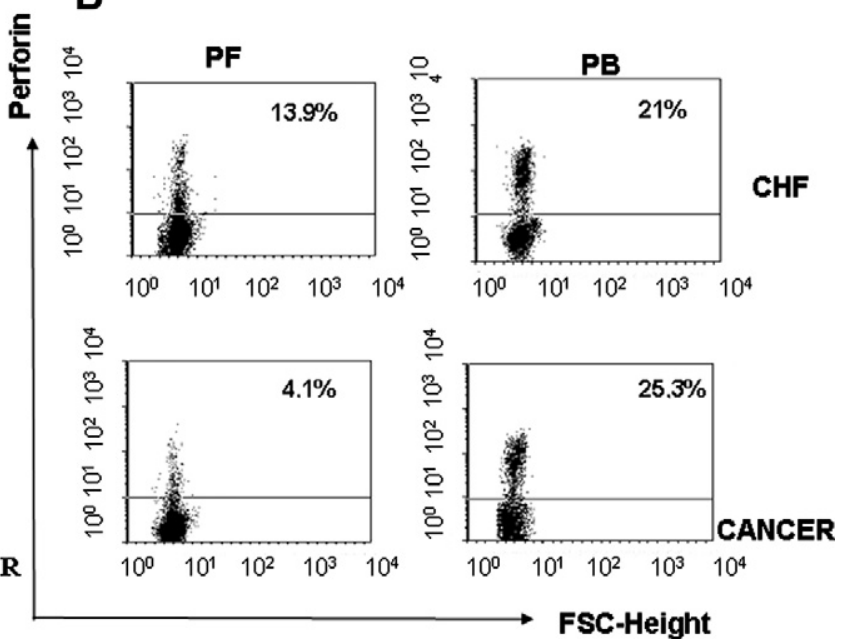

Fig. 4 - Perforin expression in cancer and CHF patients. Pleural and peripheral blood mononuclear cells were isolated from malignant $(n=19)$ and from CHF $(n=11)$ pleural fluids and perforin expression was assessed by flow-cytometry in the lymphocytes gated by forward and side scatter (see Section for details) using a FITC conjugated antibody. Data are expressed as percentage of positive cells (mean \pm S.D(A). * $p<0.05$ (Mann Whitney $U$-test); ${ }^{* *} p<0.05$ (paired t test). (B) Representative dot plots that show the percentage of total lymphocytes expressing perforin. 
A

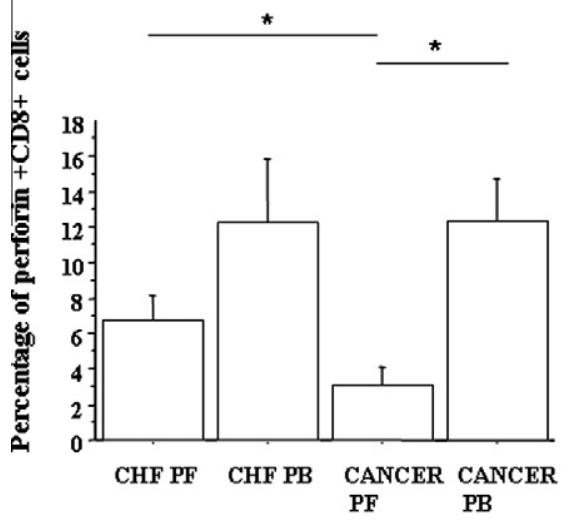

B
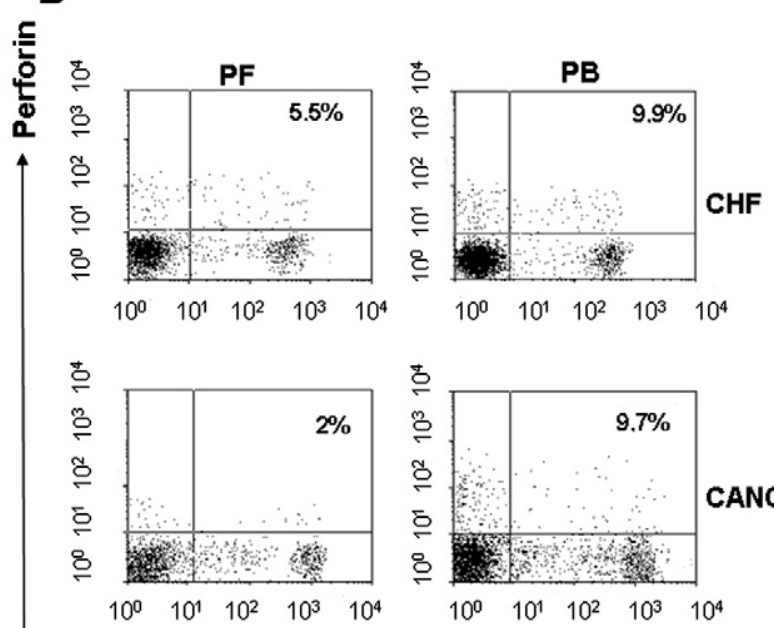

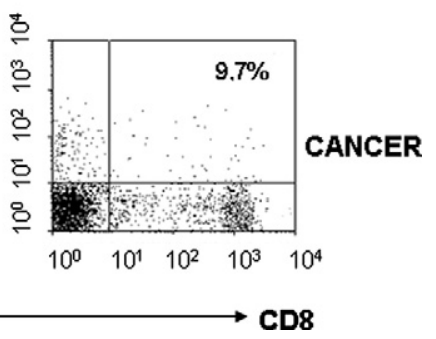

Fig. 5 - Perforin expression in CD8+ cells from cancer and CHF patients. Pleural and peripheral blood mononuclear cells were isolated from malignant $(n=19)$ and from CHF $(n=11)$ pleural fluids and perforin expression in CD8+ cells was assessed by flow-cytometry in the lymphocytes gated by forward and side scatter (see Section for details) using a FITC conjugated antibody for perforin and a PE conjugated antibody or CD8. Data are expressed as percentage of positive cells (mean \pm S.D.) (A). * $p<0.05$ (Mann Whitney U-test). (B) Representative dot plots that show the percentage of CD8+ cells expressing perforin.

cells and represent a suitable model for the study of interactions between tumour cells and the host immune system. Although, it is well known that specific cancers, such as lung cancer or cancer of the breast, ovary and stomach preferentially metastasise the pleural compartment, ${ }^{1}$ the mechanisms that promote a predilection for the pleural compartment are not fully elucidated. We previously demonstrated that pleural inflammation generates an ideal microenvironment to attract cancer cells, to promote their growth and their protection when exposed to toxic agents. ${ }^{2}$ The present study was aimed at extending these findings by investigating whether the recruitment and the accumulation of specific subsets of lymphocytes in the malignant effusions may contribute to these cancer protective events within the pleural compartment.

We have found that pleural CD8+ CTL from cancer patients show a reduced expression of CD94/NKG2A when compared to autologous $\mathrm{PB}$ and to $\mathrm{CHF} \mathrm{PF}$ and a reduced expression of perforin when compared to autologous PB.
A

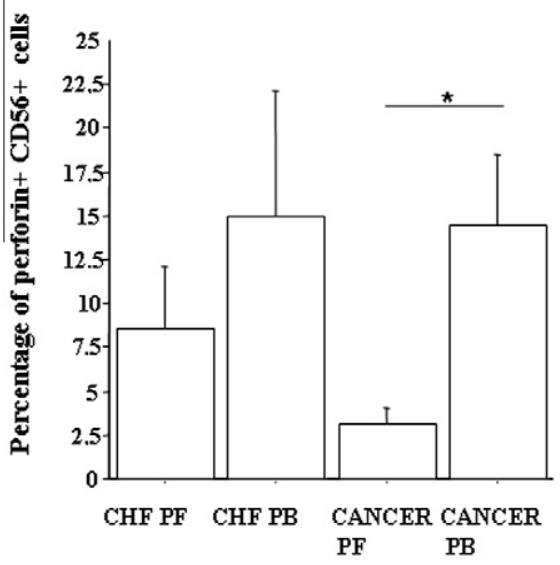

B
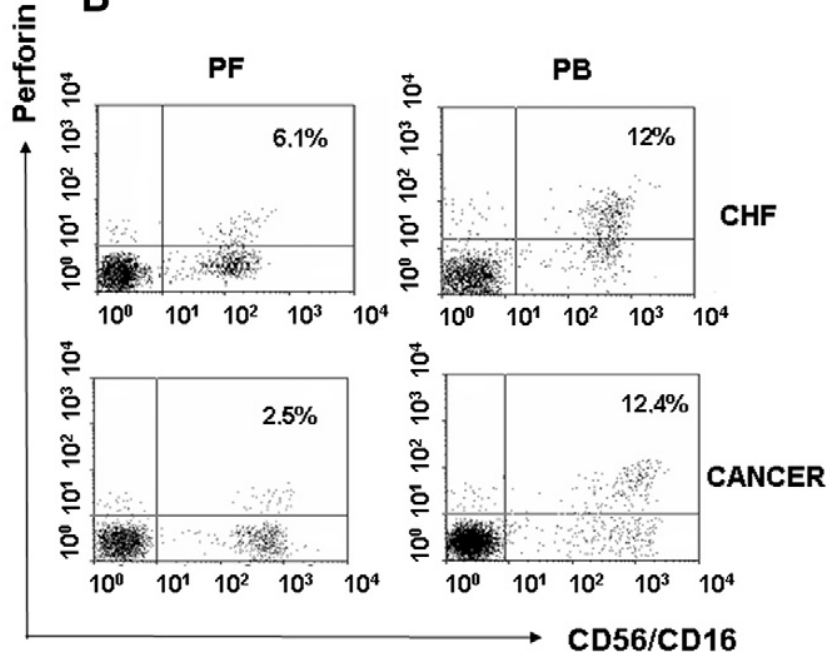

Fig. 6 - Perforin expression in CD56+/CD16+ cells from cancer and CHF patients. Pleural and peripheral blood mononuclear cells were isolated from malignant $(n=19)$ and from CHF $(n=11)$ pleural fluids and perforin expression in double positive CD16+/CD56+ (NK) cells was assessed by flow-cytometry in the lymphocytes gated by forward and side scatter (see Section for details) using a FITC conjugated antibody for perforin and a PE-anti CD16 and PE-Cy5 anti-CD56 conjugated antibodies. Data are expressed as percentage of positive cells (mean \pm S.D.) (A). ${ }^{*} p<0.05$ (Mann Whitney $U$-test). (B) Representative dot plots that show the percentage of CD56/CD16+ cells expressing perforin. 

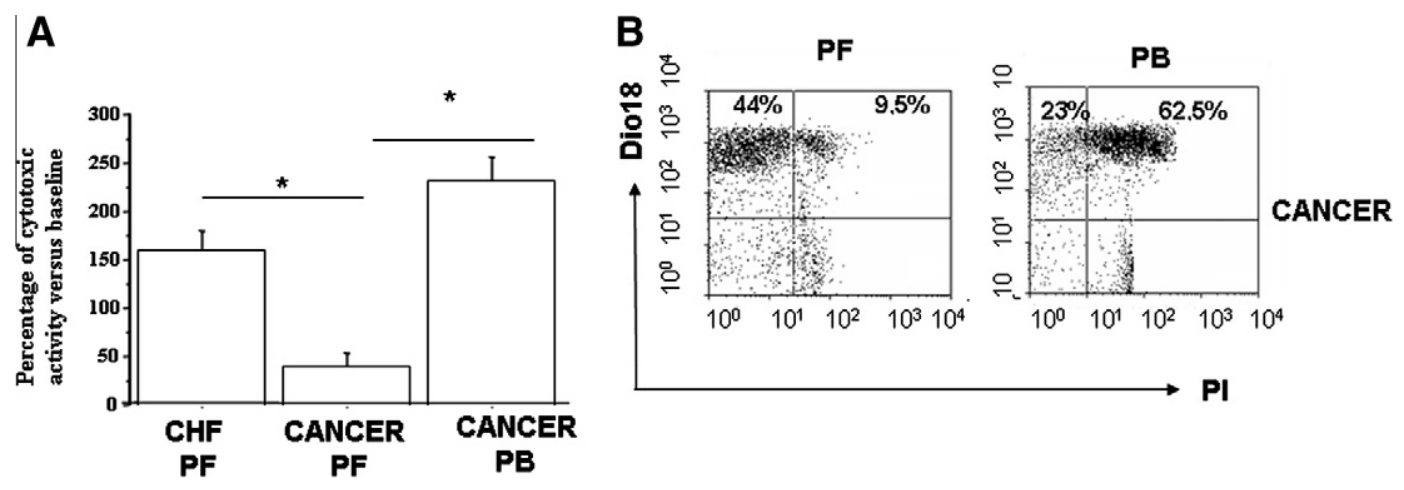

Fig. 7 - Cytotoxic activity against cancer cells. Pleural and peripheral blood mononuclear cells and pleural mononuclear cells were isolated from cancer $(n=8)$ and from $\mathrm{CHF}(n=8)$ patients, respectively and a flow cytotoxic assay against H292 cancer cells was performed using the DiO membrane dye and propidium iodide (PI) (see Section for details). Data are expressed as percentage of cytotoxic activity versus baseline (=H292 cultured in medium alone) (A). ${ }^{*} p<0.05$ (paired $t$ test). (B) Representative dot plots from a cancer patient that show the percentage of DiO positive (cancer cells) or negative cells expressing PI.

Reduced numbers of NK are present in PF from both cancer and CHF patients, and these cells in malignant PF have a reduced expression of membrane CD94/NKG2A and of perforin in comparison to autologous PB. Moreover, pleural lymphocytes from cancer patients with higher intracellular CD94/ NKG2A expression exhibit a reduced cytotoxic activity against cancer cells when compared to autologous PB T lymphocytes and to CHF PF.

CD94/NKG2A is expressed on NK cells and on CD8+ CTL. The CD94/NKG2 heterodimer is a C-type lectin receptor, formed by the covalent association of CD94, a protein with a short non-signalling intracytoplasmic tail and one of the NKG2 molecules, namely NKG2A, -B, -C or -E. The intracellular domain of NKG2A contains immunoreceptor tyrosinebased inhibition motifs (ITIMs), responsible for transducing inhibitory signals, useful to limit exaggerated activation of NK cells and of CD8+ CTL. In humans, CD94/NKG2A is activated upon the interaction with complexes of non-classical HLA-E molecules. ${ }^{11}$ Consequently, the modulation of the ligands of CD94/NKG2A on the surface of cancer cells may affect the functional activation of effector cells.

CD8+ CTL play an important role in cell-mediated immune responses. They are capable of inducing the death of cancer cells by the release of perforin and granulysin. Perforin forms pores in the plasma membrane of target cell allowing granzymes types of serine proteases, to enter the target cell, which then activates a series of enzymes, the caspase cascade that eventually lead to apoptosis. ${ }^{13}$

Human NK cells are generally recognised as sentinels of the innate immune system due to their inherent capacity to deal with diseased (stressed) cells, including malignant cells. ${ }^{14}$ More than $90 \%$ of circulating NK cells express moderate levels of CD56 (CD56dim NK cells) and high levels of CD16 and are heterogeneous in the expression of CD94 and NKG2A molecules.

The minor subset of NK cells expresses high levels of CD56 (CD56bright), CD94 and NKG2A, tends to lack the expression of $\mathrm{CD} 16^{15}$ and have been found to be enriched in human secondary lymphoid organs and in chronic inflammatory sites. ${ }^{16}$
NK infiltrating human non-small lung cancer are characterised by the high expression of CD56 and have a reduced ability to kill cancer cells. ${ }^{17}$

In our study, a significant reduction of membrane CD94/ NKG2A expression and of perforin was observed in CD8+ CTL and in NK cells isolated from malignant PF in comparison to those isolated from autologous PB. Furthermore, a significant reduction of membrane CD94/NKG2A expression and of perforin was observed in CD8+ CTL isolated from malignant PF in comparison to those isolated from CHF PF, suggesting that these changes in the phenotype of the recruited cells may be generated by the presence of an inflammatory milieu into the pleural compartment. This finding is specific for exudative malignant effusions since in exudative tuberculous PF there is an accumulation of subsets of NK cells that are characterised by low expression of perforin but higher expression of CD94/NKG2A and higher levels of IFNg in comparison to the PB counterparts. ${ }^{15}$

The distribution of $\mathrm{T}$ lymphocyte subsets in various organ compartments is very different than the distribution of subsets in the peripheral blood. This compartmentalisation is essential for optimal host defence. Pleural microenvironment (cells and mediators) tightly participates in the regulation of immune responses within the pleural space. ${ }^{18,19}$ Selective recruitment ${ }^{20-22}$ or selective cell apoptosis ${ }^{23}$ may affect the accumulation of specific cells in the pleural compartment during inflammation. Peripheral blood CD16 positive cells with low expression of CD56 are more susceptible to undergo apoptosis in tuberculosis pleural effusions leading to an enrichment in activated CD16 negative cells with high expression of CD56. ${ }^{15}$ Moreover, $\mathrm{PGE}_{2}$ released in malignant pleural effusions protect $\mathrm{T}$ lymphocyte from apoptosis and this protective effect is mainly exerted on the CD45RO+ T cells rather than on CD45RA+ T cells. ${ }^{23}$

Consistently, the majority of CD8+ T lymphocytes in the pleural compartment expresses CD45RO, ${ }^{24}$ a surface molecule that has been correlated with the memory phenotype and granzymes but not perforin or expresses perforin at a low level. ${ }^{24}$ In addition CD94 down-regulation may contribute to 

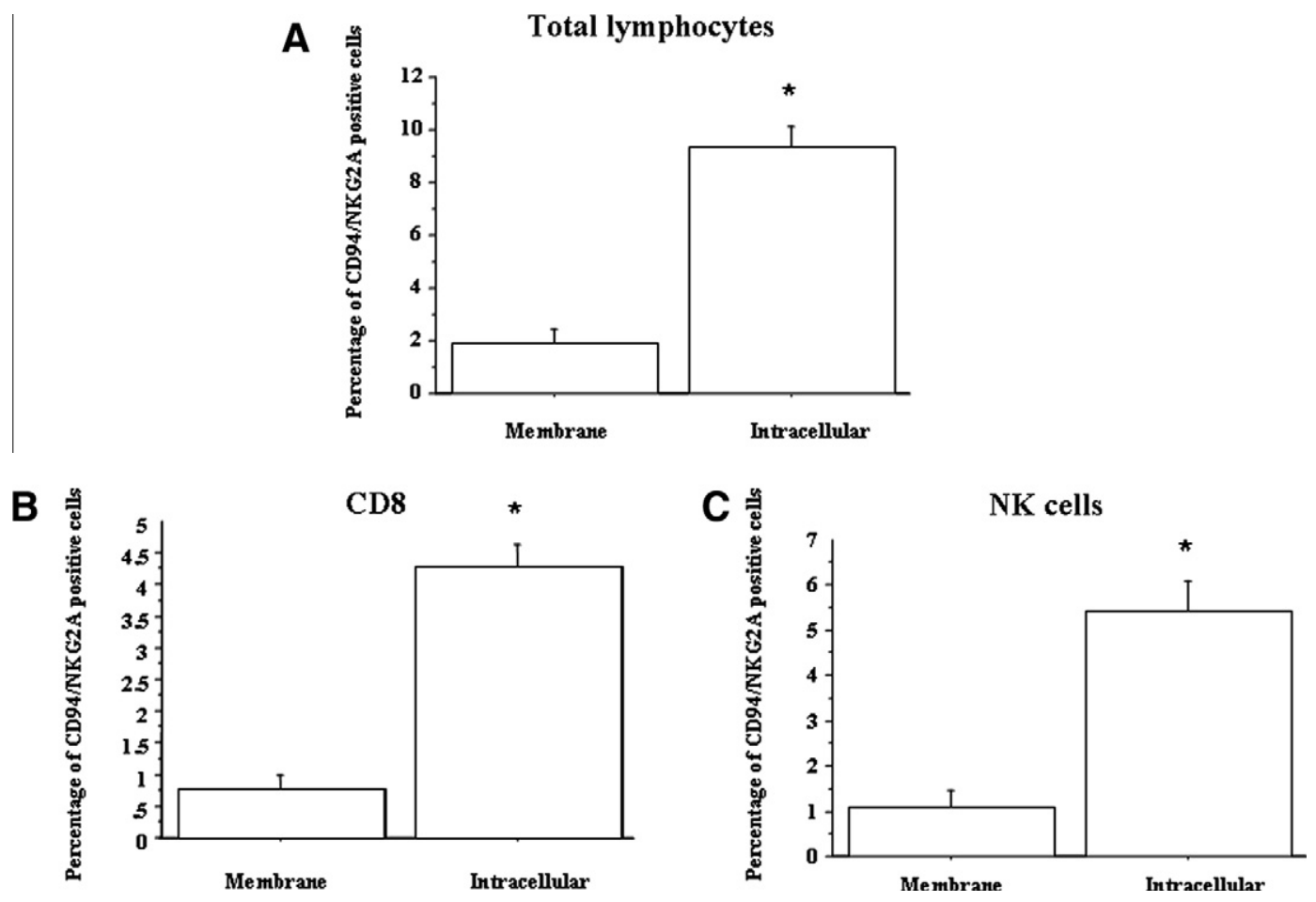

Fig. 8 - Intracellular expression of CD94/NKG2A in pleural cells from cancer patients. Pleural mononuclear cells were isolated from malignant pleural effusions $(n=10)$ and the membrane and intracellular CD94/NKG2A expression was evaluated (see Section for details). Data are expressed as percentage of CD94/NKG2A positive cells. ${ }^{*} p<0.05$ (paired $t$ test).

promote the cell apoptosis in specific cell types since its expression correlates with the survival of CD8+ CTL and NK cells. ${ }^{25} \mathrm{~A}$ preferential cell apoptosis in the terminally differentiated CD8+ CTL and NK effector cells could result in a reduced efficiency of immune responses. In this regard, down-regulation of CD94/NKG2A on CD8+ CTL and NK cells has been observed in advanced HIV infection. ${ }^{26}$ In addition, activated CD4+ $\mathrm{T}$ cells that fail to engage inhibitory NKG2A receptors on the membrane of NK cells are also susceptible to NK cell lysis. Consequently, the reduced membrane expression of NKG2A receptors in malignant PF might negatively affect the development of adaptive responses in this compartment. In this regard, it has been previously demonstrated that patients with malignant effusions show a deficient enrichment of $\mathrm{T}$ cells expressing the phenotype of type-1polarised effector $\mathrm{T}$ cells at the tumour site. ${ }^{8}$

Although, CD8+ CTL and NK cell recovered from cancer PF have a reduced expression of the inhibitory receptor CD94/ NKG2A, they exhibit a reduced cytotoxic activity against cancer cells when compared to CD8+ CTL and NK cells recovered from autologous PB. NKG2A receptor deficiency may contribute to this reduced cytotoxic activity by two mechanisms: (1) high concentrations of IFN- $\gamma$ increase the levels of HLA-E which activate the receptor thus promoting its internalisation (being the receptor no longer on the surface) ${ }^{11}$; (2) the lack of the NKG2A receptor enhances regulatory functions with an advantage for the tumour cell. In this regard, it has been demonstrated that genetic disruption of the NKG2A-Qa-1 interaction in mice releases the brakes on CD8-dependent suppressive activity, allowing the development of robust
CD8- Treg activity. ${ }^{27}$ These CD8+ cells with Treg activity also express low levels of granzyme $B$ and perforin, suggesting that these cells do not possess killing potential. ${ }^{28}$ Additionally, perforin deficiency increases the susceptibility to cancer. $^{13}$

Indeed, these concepts are consistent with our present findings, in that CD8+ CTL and NK cells from malignant PF have elevated intracellular expression of CD94/NKG2A (i.e. activated form of receptor), undetectable perforin expression and a reduced ability to lyse tumour target cells. Furthermore, it has been previously demonstrated that reduced number of NK and increased numbers of regulatory $\mathrm{T}$ cells are observed in malignant lung tissue in non-small cell lung cancer. $^{29}$

In conclusion, this study identifies new mechanisms promoting the development and persistence of a compartmentalised pleural inflammation as well as a reduced host defence mechanism against cancer within the pleural space.

\section{Conflict of interest statement}

None declared.

\section{Acknowledgements}

This work was supported by the Italian National Research Council (C.N.R.), the University of Parma, and the University of Palermo. 


\section{R E F E R E N C E S}

1. Antony VB, Loddenkemper R, Astoul P, et al. Management of malignant pleural effusions. Eur Respir J 2001;18:402-19.

2. Pace E, Siena L, Ferraro M, et al. Role of prostaglandin E2 in the invasiveness, growth and protection of cancer cells in malignant pleuritis. Eur J Cancer 2006;42:2382-9.

3. Loose $D$, Van de Wiele $C$. The immune system and cancer. Cancer Biother Radiopharm 2009;24:369-76.

4. Pardo J, Aguilo JI, Anel A, et al. The biology of cytotoxic cell granule exocytosis pathway: granzymes have evolved to induce cell death and inflammation. Microbes Infect 2009;11:452-9.

5. Chávez-Galán L, Arenas-Del Angel MC, Zenteno E, Chávez R, Lascurain R. Cell death mechanisms induced by cytotoxic lymphocytes. Cell Mol Immunol 2009;6:15-25.

6. Gunturi A, Berg RE, Forman J. The role of CD94/NKG2 in innate and adaptive immunity. Immunol Res 2004;30:29-34.

7. Borrego F, Kabat J, Sanni TB, Coligan JE. NK cell CD94/NKG2A inhibitory receptors are internalized and recycle independently of inhibitory signaling processes. J Immunol 2002;169:6102-11.

8. Atanackovic D, Block A, de Weerth A, et al. Characterization of effusion-infiltrating T cells: benign versus malignant effusions. Clin Cancer Res 2004;10:2600-8.

9. Light RW. Pleural diseases. Dis Mon 1992;38:261-331.

10. Comin CE, Novelli L, Boddi V, Paglierani M, Dini S. Calretinin, thrombomodulin, CEA, and CD15: a useful combination of immunohistochemical markers for differentiating pleural epithelial mesothelioma from peripheral pulmonary adenocarcinoma. Hum Pathol 2001;32:529-36.

11. Peruzzi G, Masilamani M, Borrego F, Coligan JE. Endocytosis as a mechanism of regulating natural killer cell function: unique endocytic and trafficking pathway for CD94/NKG2A. Immunol Res 2009;43:210-22.

12. Ruffell B, Denardo DG, Affara NI, Coussens LM. Lymphocytes in cancer development: polarization towards pro-tumor immunity. Cytokine Growth Factor Rev 2010;21:3-10.

13. Brennan AJ, Chia J, Trapani JA, Voskoboinik I. Perforin deficiency and susceptibility to cancer. Cell Death Differ 2010;17:607-15.

14. Cooper MA, Fehniger TA, Caligiuri MA. The biology of human natural killer-cell subsets. Trends Immunol 2001;22:633-40.

15. Schierloh $\mathrm{P}$, Yokobori $\mathrm{N}$, Alemán $\mathrm{M}$, et al. Increased susceptibility to apoptosis of 6dimCD16_NK cells induces the enrichment of IFN- $\gamma$ producing CD56bright cells in tuberculous pleurisy. J Immunol 2005;175:6852-60.

16. Dalbeth N, Gundle R, Davies RJ, et al. CD56bright NK cells are enriched at inflammatory sites and can engage with monocytes in a reciprocal program of activation. J Immunol 2004;173:6418-26.
17. Carrega P, Morandi B, Costa R, et al. Natural killer cells infiltrating human nonsmall-cell lung cancer are enriched in CD56 bright CD16(-) cells and display an impaired capability to kill tumor cells. Cancer 2008;112:863-75.

18. Pace E, Ferraro M, Mody CH, et al. Pleural mesothelial cells express both BLT2 and PPARa and mount an integrated response to pleural $\mathrm{LTB}_{4}$. J Immunol 2008;181:7292-9.

19. Melis M, Pace E, Siena L, et al. Biologically active intercellular adhesion molecule-1 is shed as dimers by a regulated mechanism in the inflamed pleural space. Am J Respir Crit Care Med 2003;167:1131-8.

20. Pace $\mathrm{E}$, Gjomarkaj $\mathrm{M}$, Melis $\mathrm{M}$, et al. Interleukin-8 induces lymphocyte chemotaxis within the pleural space that is regulated by pleural macrophages. Am J Respir Crit Care Med 1999;159:1592-9.

21. Pace E, Profita M, Melis M, et al. $\mathrm{LTB}_{4}$ is present in exudative pleural effusions and actively contributes to neutrophil recruitment in the inflamed pleural space. Clin Exp Immunol 2004;135:519-27.

22. Weninger W, Crowley MA, Manjunath N, von Andrian UH. Migratory properties of naive, effector, and memory CD8 C T cells. J Exp Med 2001;194:953-66.

23. Pace E, Bruno TF, Mody CH, et al. Elevated expression of EP2 receptor and increased release of $\mathrm{PGE}_{2}$ maintain the survival of CD45RO+ T cells in the inflamed human pleural space. Immunology 2007;121:427-36.

24. Prado-Garcia H, Aguilar-Cazares D, Flores-Vergara H, Mandoki JJ, Lopez-Gonzalez JS. Effector, memory and naïve CD8+ T cells in peripheral blood and pleural effusion from lung adenocarcinoma patients. Lung Cancer 2005;47:361-71.

25. Gunturi A, Berg RE, Forman J. Preferential survival of CD8 T and NK cell expressing high levels of CD94. J Immunol 2003;170:1737-45.

26. Zeddou M, Rahmouni S, Vandamme A, et al. Downregulation of CD94/NKG2A inhibitory receptors on CD8+ T cells in HIV infection is more pronounced in subjects with detected viral load than in their aviraemic counterparts. Retrovirology 2007;4:72.

27. Lu L, Kim HJ, Werneck MB, Cantor H. Regulation of CD8+ regulatory T cells: interruption of the NKG2A-Qa-1 interaction allows robust suppressive activity and resolution of autoimmune disease. Proc Natl Acad Sci USA 2008;105: 19420-5.

28. Nigam P, Velu V, Kannanganat S, et al. Expansion of FOXP3+ CD8 $\mathrm{T}$ cells with suppressive potential in colorectal mucosa following a pathogenic simian immunodeficiency virus infection correlates with diminished antiviral $\mathrm{T}$ cell response and viral control. J Immunol 2010;184:1690-701.

29. Esendagli G, Bruderek K, Goldmann T, et al. Malignant and non-malignant lung tissue areas are differentially populated by natural killer cells and regulatory $\mathrm{T}$ cells in non-small cell lung cancer. Lung Cancer 2008;59:32-40. 